\title{
Low-temperature quenching of one-dimensional localised Frenkel excitons
}

\author{
A. V. Malyshev \& V. A. Malyshev甘 and F. Domínguez-Adame \\ GISC, Departamento de Física de Materiales, Universidad Complutense, E-28040 Madrid, Spain
}

(Dated: July 23, 2013)

\begin{abstract}
We present a theoretical analysis of low-temperature quenching of one-dimensional Frenkel excitons that are localised by moderate on-site (diagonal) uncorrelated disorder. Exciton diffusion is considered as an incoherent hopping over localization segments and is probed by the exciton fluorescence quenching at point traps. The rate equation is used to calculate the temperature dependence of the exciton quenching. The activation temperature of the diffusion is found to be of the order of the width of the exciton absorption band. We demonstrate that the intra-segment scattering is extremely important for the exciton diffusion. We discuss also experimental data on the fast exciton-exciton annihilation in linear molecular aggregates at low temperatures.

PACS numbers: 71.35.Aa; 78.30.Ly
\end{abstract}

\section{INTRODUCTION}

Since the seminal works by Jelley [1] and Scheibe [2], the concept of Frenkel excitons [3, 4] has been admitted for the explanation of the remarkable optical properties of molecular aggregates. The most surprising among them are the appearance of a narrow and intense line in the red-wing of the absorption spectra (J-band) and the increase of the oscillator strength of the optical transition at cryogenic temperatures by almost two orders of magnitude [8, 9, 10]. During the nineties, a significant progress in understanding of optical dynamics in J-aggregates was achieved (for details see the recent reviews 5, 6, 7 and references therein).

In the recent paper 11, the anomalously fast lowtemperature mobility of excitons in linear molecular aggregates was reported. The authors of Ref. 11 studied the exciton-exciton annihilation in aggregates of the triethylthiacarbocyanine salt of 3,3'-bis(sulfopropyl)-5,5'dichloro-9-ethylthiacarbocyanine (THIATS). The process of annihilation was found to activate at low temperatures, $T<20 \mathrm{~K}$ (which is small compared to the width of the J-band, $82 \mathrm{~cm}^{-1}$ ) and, what is more important, at extremely low concentrations of excitons. This finding implies that excitons can move at low temperatures over large distances.

A great deal of theoretical work has also been devoted to description of the optical properties of J-aggregates [7]. Surprisingly, the simplest one-dimensional (1D) tightbinding model with on-site (diagonal) disorder provides a good basis for understanding complex optical dynamics in these systems. The goal of the present paper is to study whether this model describes the experimentally observed high mobility of 1D Frenkel excitons in J-aggregates at low-temperatures. To the best of our

\footnotetext{
* On leave from Ioffe Physiko-Technical Institute, 26 Politechnicheskaya str., 194021 Saint-Petersburg, Russia

†On leave from "S.I. Vavilov State Optical Institute", SaintPetersburg, Russia
}

knowledge, this problem has not been discussed in the literature yet. We use the effect of quenching of the exciton fluorescence to probe the exciton mobility. The term "low temperature" refers to the temperatures lower or of the order of the magnitude of the J-band width. Higher temperatures are beyond the scope of this paper.

The paper is organized as follows. In Sec. III we present our microscopic model. In Sec. IIII the low-temperature exciton diffusion over manifolds of the localised states and quenching are discussed qualitatively. The results of numerical simulations of the exciton fluorescence quenching, obtained on the basis of the rate equation approach, are the contents of Sec. IV In Sec. $\nabla$ we discuss the experimental data on the exciton-exciton annihilation in THIATS aggregates. Section VI concludes the paper with a summary of the main results.

\section{DESCRIPTION OF THE MODEL}

We model a J-aggregate by $N(N \gg 1)$ optically active two-level molecules forming a regular in space 1D open chain. The corresponding Frenkel exciton Hamiltonian reads [4] (for the sake of simplicity only the nearestneighbor interaction is considered)

$$
H=\sum_{n=1}^{N} E_{n}|n\rangle\langle n|-J \sum_{n=1}^{N-1}(|n+1\rangle\langle n|+| n\rangle\langle n+1|) .
$$

Here $E_{n}$ is the excitation energy of the $n$-th molecule, $|n\rangle$ denotes the state vector of the $n$-th excited molecule. The energies $E_{n}$ are assumed to be Gaussian uncorrelated (for different sites) stochastic variables distributed around the mean value $\omega_{0}$ (which is set to zero without loosing generality) with the standard deviation $\Delta$. The hopping integral, $-J$, is considered to be non-random and negative $(J>0)$, which corresponds to the case of J-aggregates (see, e.g., Ref. [8]). In this case the states coupled to the light are those close to the bottom of the exciton band. We consider moderate disorder $(\Delta<J)$ in what follows. This implies that the exciton eigenstates 
$\varphi_{\nu}(\nu=1,2, \ldots, N)$ found from

$$
\sum_{n=1}^{N} H_{n m} \varphi_{\nu m}=\varepsilon_{\nu} \varphi_{\nu n}, \quad H_{n m}=\langle n|H| m\rangle
$$

are extended over relatively large segments of the chain. However, the typical size of these localisation segments, $N^{*}$, is small compared to the chain length $N$.

Having been excited into an eigenstate $\nu$, an exciton cannot hop to other eigenstates if coupling to vibrations is not taken into account. This coupling causes the incoherent hopping of excitons from one eigenstate to another. We take the hopping rate from the state $\nu$ to the state $\mu$ in the following form (see, e.g., Ref. 12)

$$
\begin{aligned}
W_{\mu \nu}=W_{0} S\left(\left|\varepsilon_{\nu}-\varepsilon_{\mu}\right|\right) \sum_{n=1}^{N} \varphi_{\nu n}^{2} \varphi_{n \mu}^{2} & \\
& \times\left\{\begin{array}{ll}
n\left(\varepsilon_{\mu}-\varepsilon_{\nu}\right), & \varepsilon_{\mu}>\varepsilon_{\nu} \\
1+n\left(\varepsilon_{\nu}-\varepsilon_{\mu}\right), & \varepsilon_{\mu}<\varepsilon_{\nu}
\end{array} .\right.
\end{aligned}
$$

Here, the constant $W_{0}$ characterizes the amplitude of the hopping and $n(\varepsilon)=[\exp (\varepsilon / T)-1]^{-1}$ is the occupation number of the vibration mode with the energy $\varepsilon$ (the Boltzmann constant is set to unity). Due to the presence of the $n(\varepsilon)$ and $1+n(\varepsilon)$ factors, the rate $W_{\mu \nu}$ meets the principle of detailed balance: $W_{\mu \nu}=$ $W_{\nu \mu} \exp \left[\left(\varepsilon_{\nu}-\varepsilon_{\mu}\right) / T\right]$. Thus, in the absence of decay channels, the eventual exciton distribution is the Boltzmann equilibrium distribution. The sum over sites in (3) represents the overlap integral of exciton probabilities for the states $\mu$ and $\nu$. The spectral factor $S\left(\left|\varepsilon_{\nu}-\varepsilon_{\mu}\right|\right)$ depends strongly on the particular details of the excitonphonon coupling as well as on the density of states of the medium into which the aggregate is embedded. The study of these details is beyond the scope of the present paper. We use the linear form $S\left(\left|\varepsilon_{\nu}-\varepsilon_{\mu}\right|\right)=\left|\varepsilon_{\nu}-\varepsilon_{\mu}\right| / J$ of this factor, which accounts for the reduction of the hopping in the long-wave acoustic limit [14]. The hopping rate in the form of Eq. (3) was used in Ref. 13, 14] to successfully describe the $1 \mathrm{D}$ exciton thermalization.

The mobility of excitons can be probed by the quenching of the exciton fluorescence, which is due to point traps. The quenching rate of the exciton state $\nu$ is assumed to be proportional to the probability of finding the exciton at trap sites

$$
\Gamma_{\nu}=\Gamma \sum_{i=1}^{N_{q}}\left|\varphi_{\nu i}\right|^{2},
$$

where $\Gamma$ is the amplitude of exciton quenching and the sum runs over positions of the $N_{q}$ traps. We assume also that point traps do not change neither the disorder configuration nor the exciton eigenfunctions $\varphi_{\nu i}$.

We describe the process of the exciton trapping by means of the rate equation:

$$
\dot{P}_{\nu}=-\left(\gamma_{\nu}+\Gamma_{\nu}\right) P_{\nu}+\sum_{\mu=1}^{N}\left(W_{\nu \mu} P_{\mu}-W_{\mu \nu} P_{\nu}\right)
$$

where $P_{\nu}$ is the population of the $\nu$ th exciton eigenstate and the dot denotes the time derivative, $\gamma_{\nu}=$ $\gamma\left(\sum_{n=1}^{N} \varphi_{\nu n}\right)^{2}$ is the spontaneous emission rate of the $\nu$ th exciton state, while $\gamma$ is that of a monomer. The initial total population is normalized to unity: $\sum_{\nu} P_{\nu}(0)=1$.

The temperature dependence of the exciton quenching is calculated as follows. We admit the definition of the exciton fluorescence decay time $\tau$ as the total population integrated over time (see, for instance, Ref. 15]). In the presence of disorder, it has to be averaged over disorder configurations and trap positions. Then, the equation for $\tau$ reads

$$
\begin{aligned}
\tau=\int_{0}^{\infty} d t \int d E\left\langle\sum_{\nu=1}^{N} \delta\left(E-E_{\nu}\right) P_{\nu}(t)\right\rangle= \\
=\int_{0}^{\infty} d t\left\langle\sum_{\nu=1}^{N} P_{\nu}(t)\right\rangle
\end{aligned}
$$

where angle brackets denote averaging. The decay time is calculated for aggregates with traps (denote it as $\tau$ ) and without traps (denote it as $\tau_{0}$ ) for the same set of disorder configurations. Recall that, according our assumption, traps do not change the exciton eigenfunctions. The quenching rate is then defined as

$$
W_{q}=\frac{1}{\tau}-\frac{1}{\tau_{0}} .
$$

This quantity carries information about the diffusion rate and is the object of our analysis.

The definition of the decay rate as the integrated total population allows for considerable simplification of the calculation procedure. We write the solution of Eq. (5) in a formal matrix form

$$
P_{\nu}=\sum_{\mu=1}^{N}\left(e^{-\hat{R} t}\right)_{\nu \mu} P_{\mu}(0),
$$

where

$$
R_{\nu \mu}=\left(\gamma_{\nu}+\Gamma_{\nu}+\sum_{\mu=1}^{N} W_{\mu \nu}\right) \delta_{\mu \nu}-W_{\nu \mu} .
$$

After the substitution of (8) into Eq. (6) and integration over time, $\tau$ can be expressed in terms of the $\hat{R}$-matrix:

$$
\tau=\left\langle\sum_{\nu, \mu=1}^{N}\left(\hat{R}^{-1}\right)_{\nu \mu} P_{\mu}(0)\right\rangle .
$$

Clearly, obtaining $W_{q}$ only requires the calculation of the inverse matrix $\hat{R}^{-1}$ for each realization of disorder rather than the tedious calculation of the kinetics. The inverse matrix is to be found twice: for an aggregate with and without traps. Note that the decay time in the absence of traps, $\tau_{0}$, also depends on temperature (see, for example, Ref. [14]). 


\section{QUALITATIVE PICTURE}

At low temperatures, excitons occupy states at the bottom of the exciton band. Therefore, this part of the exciton energy spectrum determines the low-temperature exciton transport. Below, we recall briefly the concept of local (hidden) energy structure of localised 1D excitons 16, 17, 19], which was proved to exist in the vicinity of the band bottom [20, 21]. According to this concept, the low-energy one-exciton eigenfunctions obtained for a fixed realization of disorder can be grouped into local manifolds of two (or sometimes more) states that are localised at the same chain segment of typical length $N^{*}$ (in units of the lattice constant) which scales with disorder as follows 20 .

$$
N^{*}=8.71\left(\frac{\Delta}{J}\right)^{-0.67}
$$

It turns out that the structure of the exciton states in each local manifold is very similar to the structure of the lower states of a regular (non-disordered) chain of length $N^{*}$. In particular, the lowest state in a manifold has a wave function without nodes within its localisation segment. Such a state can be interpreted as the local ground state of the segment. It carries large oscillator strength, approximately $N^{*}$ times larger than that of a monomer, so that the typical spontaneous emission rate is $\gamma^{*}=\gamma N^{*}$. The second state in the manifold has a node within the localisation segment and looks like the first local excited state of the segment (see the states filled with black color in Fig. 11). Its oscillator strength is typically an order of magnitude smaller than that of the local ground state. It is important to note that, contrary to the eigenstates from the same manifold, the states from different manifolds overlap weakly. The energies of local ground states are distributed within the interval $\sigma_{11}$ that is larger than the typical energy spacing $\varepsilon_{12}$ between the levels in a local manifold [20]:

$$
\begin{aligned}
& \sigma_{11}=0.67 \mathrm{~J}\left(\frac{\Delta}{J}\right)^{1.33}, \\
& \varepsilon_{12}=0.40 \mathrm{~J}\left(\frac{\Delta}{J}\right)^{1.36} .
\end{aligned}
$$

For this reason, the local energy structure cannot be seen either in the density of states (DOS) or in the linear absorption spectra. However, it determines the nonlinear optical response of the system 17, 22, 23, 24].

Higher states are more extended than the local states as the localisation length increases with energy. Therefore, the higher states cannot be included into any particular local manifold: their wave functions cover more than one $N^{*}$-molecule segments (see the states filled with gray color in Fig. 1). The typical energy spacing between these higher states and the covered local states is of the

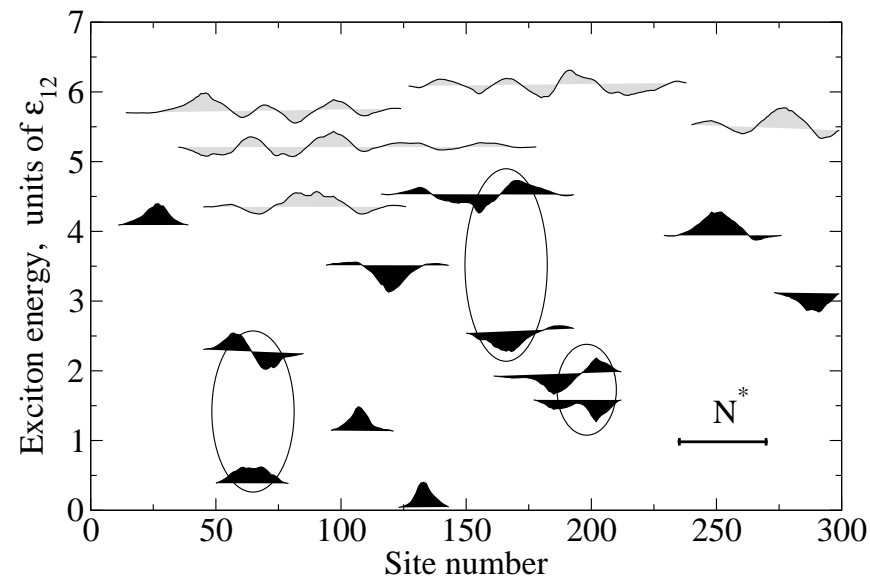

FIG. 1: The energy structure of the exciton levels in the vicinity of the bottom of the exciton band. The states are obtained by diagonalization of the Hamiltonian (1) for a linear chain of 300 sites and the disorder magnitude $\Delta=0.1 \mathrm{~J}$. The baseline of each state represents its energy in units of $\varepsilon_{21}$. The origin of the exciton energy is set to the lowest energy for the realization. The wave functions are in arbitrary units. It is clearly seen that the lower states can be grouped into local manifolds. The states within each such manifold are localised at the same segment; they overlap well with each other and overlap much weaker with the states of other manifolds.

order of $\varepsilon_{12}$ Thus, the energy $\varepsilon_{12}$ is expected to be the activation energy for the exciton diffusion.

It is clear from the above arguments that the energy structure of the lower exciton states is of importance for understanding the features of the low-temperature exciton transport. On the basis of the local energy structure concept, two types of exciton hopping over the local states can be distinguished: intra-segment hopping and inter-segment one, involving the states of the same local manifold and of different manifolds, respectively. As the states from different local manifolds overlap weakly (see Fig. 11), only inter-segment hops to adjacent segments are of importance. The disorder scaling of the overlap integrals $I_{\mu \nu}=\sum_{n} \varphi_{\mu n}^{2} \varphi_{\nu n}^{2}$ for the local states of the same and adjacent segments was obtained in Ref. 21:

$$
\begin{gathered}
I_{12}=0.14\left(\frac{\Delta}{J}\right)^{0.70} \\
I_{\nu^{\prime} 1}=I_{\nu^{\prime} 2} \approx 0.0025\left(\frac{\Delta}{J}\right)^{0.75} .
\end{gathered}
$$

Hereafter, the indices 1 and 2 label the local states of the same segment while those with primes label the local states of a different adjacent segment. As follows from Eqs. (13a)-13b), the intra-segment overlap integral is typically more than an order of magnitude larger than the inter-segment one. The intra-segment hops do not result in the spatial displacement of excitons; they correspond to the intra-segment relaxation. Only the 
inter-segment hopping gives rise to the spatial motion of excitons. Nevertheless, we show that both types of hops are important for understanding the features of the lowtemperature exciton transport.

The overlap integrals between the local states of a segment and the higher states which are extended over this segment are of the order of $I_{12}$. This fact implies that even at $T<\varepsilon_{12}$, the hops via these higher states can be more efficient than the inter-segment hops over the local states.

\section{A. Zero temperature}

At zero temperature an exciton can hop only down to lower states. Let us assume that it is in the local excited state 2 . Then it can only hop to the local ground state of the same segment 1 or to a lower state $\nu^{\prime}$ localised at an adjacent segment (see Fig. 2 $T=0$ ). Because the intra-segment hopping is faster than the intersegment one, first, the exciton hops down to the local ground state 1 with the typical energy loss $\varepsilon_{12}\left(\varepsilon_{12}\right.$ being the mean energy spacing in the local discrete energy structure, see Fig. 2] $T=0$ ). From the local ground state, the exciton can hop only to a state $\nu^{\prime}$ of an adjacent segment provided that $\varepsilon_{\nu^{\prime}}<\varepsilon_{1}$ and the spontaneous emission rate of the local ground state $\gamma_{1}$ is small compared to the intra-segment hopping rate $\left.W_{\nu^{\prime} 1}\right|_{T=0}$. Hereafter, such a relationship between these rates is referred to as the limit of fast diffusion; only this limit is considered in this work. The typical energy loss during such sideways hop is of the order of the width of the local ground states distribution, $\sigma_{11}\left(\sigma_{11}\right.$ is about the J-band width). Thus, already after one such sideways hop the exciton resides in a state in the tail of the DOS. Therefore, the number of states with even lower energies decreases dramatically, which results in a strong increase in the typical distance to those states and in a suppression of the probability to hop further sideways. Then the exciton either relaxes to a lower state of the same segment (if there is one) or decays spontaneously, i.e., this type of the spatio-energetic diffusion (towards lower energies) stops very quickly. Note that this diffusion would manifest itself in the red shift of the exciton emission spectrum relative to the absorption spectrum. The experimental data show that such red shift is either absent [8, 9] or is smaller than the J-band width. 11, 25]. These experimental findings unambiguously indicate that at low temperatures, $T \ll \sigma_{11}$, excitons make few hops before they decay due to the spontaneous emission, as was argued in Refs. 26, 27, 28. Consequently, the zerotemperature exciton quenching is expected to be weak provided the concentration of quenchers is low, the case we are interested in.
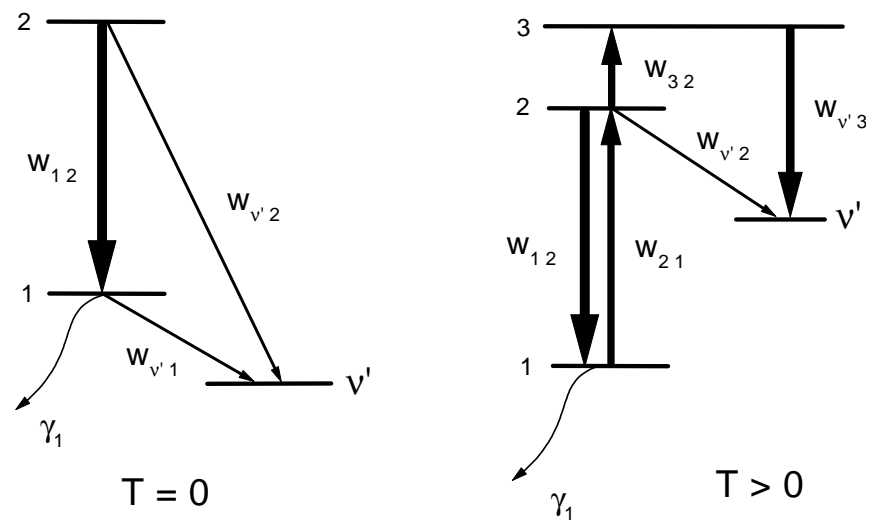

FIG. 2: Schematic view of exciton hoppings at zero and nonzero temperatures. The indices 1 and 2 label the local ground and the first local excited states of the same segment. The $\nu^{\prime}$ state is localised at an adjacent segment. The index 3 labels a higher state which overlaps well with the states of two adjacent segments. Hops are shown by the straight arrows; the arrow thickness represents the hopping rate magnitude. The spontaneous emission rate $\gamma_{1}$ is depicted by thin wavy arrows.

\section{B. Non-zero temperature}

At non-zero temperatures $\left(0<T \lesssim \varepsilon_{12}\right)$, an exciton can also hop up in energy. Consider an exciton in one of the lower states in the tail of the DOS, e.g. in the local ground state 1 (see Fig. 2] $T>0$ ). For the reasons discussed above, first, the exciton hops up to the first local excited state 2 of the same segment, provided the hopping rate for the considered temperature is larger than the spontaneous emission rate $\gamma_{1}$ of the initial state 1. During this process the exciton typically gains the energy $\varepsilon_{12}$. As $\varepsilon_{12}$ is of the order of $\sigma_{11}$ 20], already after the first hop up the exciton leaves the tail of the DOS and, hence, it is likely to have a lower state $\nu^{\prime}$ localised at an adjacent segment. A hop down to this state with loss in energy is favorable and results in the spatial displacement of the exciton, i.e., in the exciton diffusion. We stress that although only sideways hops result in the spatial displacement of the exciton, it is the initial hop up from the local ground state 1 to the local excited state 2 that triggers the diffusion.

Another way for the exciton to hop sideways to the state $\nu^{\prime}$ is via the higher state 3 that overlaps well with both states 2 and $\nu^{\prime}$ (see Fig. 2$] T>0$ ). As it has been mentioned, such hops compete with the sideways hops over the local states; although the hop up to the state 3 is thermally unfavorably, the overlap integral for this hop, $I_{31}$, is large compared to that for an inter-segment hop, $I_{\nu^{\prime} 1}$. We show later that this channel of diffusion becomes efficient indeed even at relatively low temperature. 


\section{NUMERICAL RESULTS AND DISCUSSION}

In this section, we discuss the results of numerical calculation of the quenching rate $W_{q}$. In this paper, we consider the initial condition where the leftmost local ground state is excited while a single trap is located in the center of the localisation segment of the rightmost local ground state. In this case, the exciton quenching is most affected by diffusion, as the created exciton has to travel over almost the whole chain to be quenched. Thus, the exciton quenching at low concentration of traps can be studied. The quenching rate was calculated as described in section III for the parameter set corresponding to the limit of fast diffusion and effective quenching (the later limit is defined below). In all calculations we set $W_{0}=1$ and choose the parameter $\gamma$ so that the typical intersegment down-hopping rate $\left.W_{1^{\prime} 1}\right|_{T=0} \sim W_{0}\left(\sigma_{11} / J\right) I_{\nu^{\prime} 1}$ is large compared to the typical spontaneous emission rate of a local ground state $\gamma^{*}$ (the limit of fast diffusion). $\Gamma$ is chosen so that the typical quenching rate $\Gamma^{*}$ is greater than the typical intra-segment down-hopping rate $\left.W_{12}\right|_{T=0} \sim W_{0}\left(\varepsilon_{12} / J\right) I_{12}$ (the limit of effective quenching). This ensures that once an exciton hops to a local state of the segment with the trap, it is quenched almost instantaneously. More specifically, for each magnitude of the disorder $\left.W_{1^{\prime} 1}\right|_{T=0}=10 \gamma^{*}$ and $\Gamma^{*}=\left.10 W_{12}\right|_{T=0}$ : $\gamma=5 \times 10^{-8}$ and $\Gamma=0.2$ for $\Delta=0.1 \mathrm{~J} ; \gamma=4 \times 10^{-7}$ and $\Gamma=0.5$ for $\Delta=0.2 J ; \gamma=1 \times 10^{-6}$ and $\Gamma=0.9$ for $\Delta=0.3 J$. Calculations were performed for $N=1000$ and 100 realizations of the disorder.

Figure 3 shows the temperature dependence of the quenching rate $W_{q}$ for the parameter sets specified above. In the plot, the quenching rate is given in units of the typical exciton radiative rate $\gamma^{*}=\gamma N^{*}$. The temperature is given in units of the mean energy spacing in the local energy structure $\varepsilon_{12}$. Note that both $N^{*}$ and $\varepsilon_{12}$ depend on $\Delta$ as described by (11) and (12b). Figure 3 demonstrates very clearly that for all considered values of $\Delta$ at temperatures lower than $\varepsilon_{12}$ the quenching rate is vanishing. This indicates that at these temperatures the exciton cannot reach the quencher during its lifetime and decays due to the spontaneous emission. On the contrary, just after the temperature exceeds approximately $\varepsilon_{12}$ the quenching becomes noticeable: the exciton partly diffuses to the trap where it decays mostly due to quenching. Specifically, temperature of the order of $2 \varepsilon_{12}$ are required for the quenching to become as effective as the spontaneous emission: $W_{q} \sim \gamma^{*}=\gamma N^{*}$.

It is useful to estimate the effective sideways hopping rate $W$, which is required to reach the quenching level $W_{q} \sim \gamma^{*}$. To do this, one can consider the sequence of localization segments as an effective chain of "sites", the typical number of which is equal to the number of segments, $N_{s}=N / N^{*}$; the mean spacing between these "sites" is $N^{*}$. The exciton diffusion coefficient is then estimated as $D \sim W N^{* 2}$ (the lattice constant is set to unity). For the quenching to be as effective as the spontaneous decay, the exciton has to reach the quencher (lo-

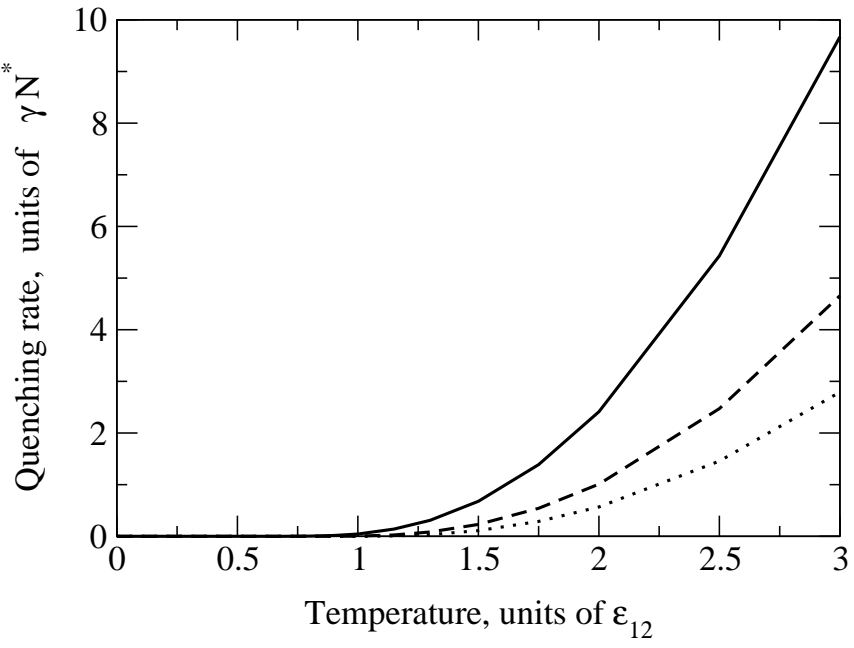

FIG. 3: Temperature dependence of the quenching rate $W_{q}$ calculated for a linear chain length $N=1000$ and different magnitudes of the disorder $\Delta$ : solid line $-\Delta=0.1 \mathrm{~J}$, dashed line $-\Delta=0.2 J$, dotted line $-\Delta=0.3 J$. The rate equation parameters were chosen so that for each magnitude of disorder $\left.W_{1^{\prime} 1}\right|_{T=0}=10 \gamma^{*}$ and $\Gamma^{*}=\left.10 W_{12}\right|_{T=0}: \gamma=5 \times 10^{-8}$ and $\Gamma=0.2$ for $\Delta=0.1 J ; \gamma=4 \times 10^{-7}$ and $\Gamma=0.5$ for $\Delta=0.2 J$; $\gamma=1 \times 10^{-6}$ and $\Gamma=0.9$ for $\Delta=0.3 \mathrm{~J}$. The averaging is performed over 100 disorder realizations. For each realization of the disorder, the leftmost local ground state is excited, while the only trap is located in the center of the localisation segment of the rightmost local ground state.

cated on the opposite side of the chain) during the lifetime $\gamma^{*-1}$, i.e., it has to diffuse over the distance $N_{s}$ during this time. Equating the diffusion length $\sqrt{D / \gamma^{*}}$ to $N$, we obtain the estimate for the required diffusion rate $W: W \sim \gamma^{*}\left(N / N^{*}\right)^{2}$. The localisation length $N^{*}$ is equal to 38,25 and 18 for $\Delta=0.1,0.2$ and 0.3 , respectively. Thus, the corresponding diffusion rates $W$ are estimated as $600 \gamma^{*}, 1600 \gamma^{*}$ and $2500 \gamma^{*}$. These values are about of two orders of magnitude larger than the rates of sideways hops over the local states, taken to be $10 \gamma^{*}$ in all calculations. This indicates that when the quenching rate becomes comparable to the spontaneous emission rate, the exciton does not hop between the local states of adjacent segments (with the typical rate $\left(W_{\nu^{\prime} 1} \sim 10 \gamma^{*}\right)$. It rather hops via the higher states that extend over more than one $N^{*}$-molecule segments (see the discussion in Sec. III). The hopping rate via such states for $T \sim 2 \varepsilon_{12}$ is of the order of $W_{12}$ which is about two orders of magnitude larger than $W_{\nu^{\prime} 1}$.

\section{ANALYZING THE FAST EXCITON-EXCITON ANNIHILATION}

In Ref. [1] the anomalously fast low-temperature diffusion of Frenkel excitons in linear aggregates of THIATS molecules was reported. The unit cell in these aggregates contains two THIATS molecules. Because of this fact, 
the absorption spectra of THIATS aggregates reveal two bands, so-called H-band and J-band 29]. The former, intensive and widely broadened (the width being about $1000 \mathrm{~cm}^{-1}$ ), results from the optical transition from the ground state of the aggregate to the top of the exciton band. The latter, much less intensive and narrower (the width being $82 \mathrm{~cm}^{-1}$ ), is due to the optical transition from the ground state to the bottom of the exciton band. Contrary to the H-band, the J-band is visible in exciton fluorescence spectra.

The authors of Ref. 11] studied the exciton-exciton annihilation in THIATS aggregates by measuring the exciton fluorescence decay after excitation into the centre of the exciton band (the whole width being about $\left.3000 \mathrm{~cm}^{-1}\right)$. It was found that this effect is pronounced even at $T=5 \mathrm{~K}\left(3.5 \mathrm{~cm}^{-1}\right)$ and at a very low intensity of excitation. In order to explain the experimental data, the authors assumed that excitons travel over about $10^{4}$ dye molecules during their lifetime to meet each other and annihilate. They found also that the activation energy for the exciton diffusion was $15 \mathrm{~K}\left(10.5 \mathrm{~cm}^{-1}\right)$ and considered this energy to be the typical energy difference between the states of adjacent localisation segments.

The fact that the exciton-exciton annihilation is very sensitive to temperature indicates that this process starts after the exciton intra-band relaxation to the states forming the J-band. Therefore, the annihilation process involves only the J-band states and the difference in band structure between THIATS aggregates and J-aggregates is probably unimportant. Furthermore, the excitonexciton annihilation can be treated similarly to the exciton quenching: one of the two excitons can be considered as an immobile trap for the other while the other diffuses twice as fast. Thus, our model is applicable to analyzing the exciton-exciton annihilation in THIATS aggregates.

As reported in Ref. [1], the fluorescence spectrum of THIATS aggregates is narrowed by approximately 26 $\mathrm{cm}^{-1}$ and experiences a red shift of $23 \mathrm{~cm}^{-1}$ as compared to the J-band. These findings indicate that the excitons make sideways hops during their lifetime, i.e., the rate of sideways hops over local states is larger than the exciton spontaneous emission rate. This indicates that the conditions for the exciton diffusion in THIATS aggregates are similar to those studied in the present paper (the limit of fast diffusion). On the basis of this analogy, the activation energy for the exciton diffusion is expected to be of the order of $82 \mathrm{~cm}^{-1}$ rather than the reported 10.5 $\mathrm{cm}^{-1}$. The typical size of localisation segment in THI-
ATS aggregates is $N^{*}=30$ [29]. In the model we are dealing with, this corresponds to the disorder magnitude $\Delta=0.2 J$. As it follows from our numerical data, the exciton quenching is vanishingly small for temperatures $T \sim(10.5 / 82) \times \varepsilon_{12}$. Thus, the model of the temperatureactivated hopping over localisation segments, proposed in Ref. 11] for the explanation of fast exciton diffusion at low temperature, is questionable. Understanding the observed fast low-temperature exciton-exciton annihilation still remains an open question.

\section{SUMMARY}

We analyzed theoretically the features of the lowtemperature diffusion of 1D Frenkel excitons localised by a moderate diagonal disorder. In this case the low-energy exciton wave functions are extended over relatively large segments of typical size $N^{*}\left(1 \ll N^{*} \ll N\right)$. We considered the exciton motion as incoherent hops over localised states. The exciton diffusion was probed by the exciton quenching by a trap that was located at one end of the chain while the exciton is initially located at the other end of the chain. For this initial condition, the quenching is most affected by the diffusion as the exciton has to travel over almost the whole chain to be quenched. Exciton quenching was described by the rate equation. Numerical simulations confirm our qualitative finding that exciton diffusion is activated at the temperature that is approximately equal to the mean spacing in the local discrete energy structure. This temperature is of the order of the J-band width. According to the general belief, at such temperatures the exciton diffuses over $N^{*}$-molecule localisation segments. We show however that it diffuses mostly over higher states which extend over few such segments. The latter provide the natural energy scale and determine the activation energy of the diffusion. We demonstrate therefore that the intra-segment scattering is extremely important for the diffusion.

\section{Acknowledgments}

This work was supported by the DGI-MCyT (Project MAT2000-0734). A. V. M. and F. D. A. acknowledge support from CAM (Project 07N/0075/2001). V. A. M. acknowledges support from MECyD (Project SAB20000103) as well as through a NATO Fellowship.
[1] E. E. Jelley, Nature (London) 38, 1009 (1936).

[2] G. Scheibe, Angew. Chem. 49, 563 (1936).

[3] J. Frenkel, Phys. Rev. 17, 17 (1931).

[4] A. S. Davydov, Theory of Molecular Excitons (Plenum, New York, 1971).

[5] F. C. Spano and J. Knoester, in Advances in Magnetic and Optical Resonance, Vol. 18, ed. W. S. Warren (Aca- demic, New York, 1994), p. 117.

[6] Contributions to Adv. Mater. 7 (1995).

[7] J-aggregates, ed. T. Kobayashi (World Scientific, Singapur, 1996).

[8] S. de Boer and D. A. Wiersma, Chem. Phys. Lett. 165, 45 (1990).

[9] H. Fidder, J. Knoester, and D. A. Wiersma, Chem. Phys. 
Lett. 171, 529 (1990).

[10] H. Fidder, J. Terpstra, and D. A. Wiersma, J. Chem. Phys. 94, 6895 (1991).

[11] I. G. Scheblykin, O. Yu. Sliusarenko, L. S. Lepnev, A. G. Vitukhnovsky, and M. Van der Auweraer, J. Phys. Chem. B 104, 10949 (2000); 105, 4636 (2001).

[12] J. A. Leegwater, J. R. Durrant, and D. R. Klug, J. Phys. Chem. B 101, 7205 (1997).

[13] M. Shimizu, S. Suto, and T. Goto, J. Chem. Phys. 114, 2775 (2001).

[14] M. Bednarz, V. A. Malyshev, J. P. Lemaistre, J. Knoester, J. Lumin. 94-95, 271 (2001).

[15] M. Bednarz, V.A. Malyshev, and J. Knoester, J. Chem. Phys. 117, 6200 (2002).

[16] V. A. Malyshev, Opt. Spektr. 71, 873 (1991) [Opt. Spectr. 71, 505 (1991)]; J. Lumin., 55, 225 (1993).

[17] V. Malyshev and P. Moreno, Phys. Rev. B 5114587 (1995).

[18] M. Shimizu, S. Suto, T. Goto, A. Watanabe, and M. Matsuda, Phys. Rev. B 58, 5032 (1998).

[19] V. A. Malyshev, A. Rodríguez, and F. DomínguezAdame, Phys. Rev. B 60, 14140 (1999a).
[20] A. V. Malyshev and V. A. Malyshev, Phys. Rev. B 63, 195111 (2001).

[21] A. V. Malyshev and V. A. Malyshev, J. Lumin. 94-95, 369 (2001).

[22] K. Minoshima, M. Taiji, K. Misawa, T. Kobayashi, Chem. Phys. Lett. 218, 67 (1994).

[23] J. Knoester and F. C. Spano, in Ref. 7, p. 111.

[24] L. D. Bakalis and J. Knoester, J. Phys. Chem. B 103, 6620 (1999); J. Lumin. 87-89, 66 (2000).

[25] V. F. Kamalov, I. A. Struganova, and K. Ioshihara, J. Phys. Chem. 100, 8640 (1996).

[26] V. A. Malyshev, H. Glaeske, and K.-H. Feller Chem. Phys. Lett. 305, 117 (1999).

[27] V. A. Malyshev, G. G. Kozlov, H. Glaeske, and K.-H. Feller. Chem. Phys. 254, 31 (2000).

[28] I. V. Ryzhov, G. G. Kozlov, V. A. Malyshev, and J. Knoester, J. Chem. Phys. 114, 5322 (2001).

[29] I. G. Scheblykin, M. M. Bataiev, M. Van der Auweraer, and A. G. Vitukhnovsky, Chem. Phys. Lett. 316, 37 (2000). 